\title{
Mobile Web Service Provisioning and Performance Evaluation of Mobile Host
}

\author{
Kishor Wagh ${ }^{1}$ and Dr. Ravindra Thool ${ }^{2}$ \\ ${ }^{1}$ Department of Computer Engineering, Pune University Marathwada Mitra Mandal's \\ Institute of Technology, Pune, India \\ ${ }^{2}$ Department of Information Technology, SRTMU SGGS College of Engineering and \\ Technology, Nanded, India
}

\begin{abstract}
Providing web services from smart phones is current recent research topic, this happened because of smart phones are used almost every area, where today's user uses for mobile banking, emailing, searching location and data. Smart phones are advanced in terms of processing power, memory with an embedded camera, sensors and same time parallel advancement in wireless network and software web technologies. Due to these advancement enables the mobile smart phones work as a web service provider instead of web service consumer. The idea of hosting web services on mobile host is not new, last one decade researchers working on mobile web service provisioning. This paper extends the our previous research work in the cellular domain to the current generation platform technologies and standards such as Android OS and REST. This paper deals with mobile host scalability and experimental result analysis for how many concurrent users access mobile host.
\end{abstract}

\section{KEYWORDS}

Smart Phone, Mobile Host, Wireless Network, Mobile Network, SOAP REST, Android

\section{INTRODUCTION}

Mobile devices (Smart phones, PDA, Tablets), Mobile web services and wireless communications, by the year 2020, it will be expect to play a central role in all aspects of lives. The Mobile web service provisioning is substantially expanding on the concept of 'anywhere, anytime and on any device' to a new paradigm ubiquitous mobile computing. It is used to improve access to meaningful, quickly and required information and content through mobile web services. Many of the problems of mobile web services can be solved by targeting the distributed nature and isolated deployment of mobile applications. One of the most promising ways to create viable web services for mobile devices is to add extra intelligence to the web services, both on the web service provider and the web service consumer.

Web services are different from web servers. Web services are the Internet-based web application that can be deployed independently and invoked by a web client browser. Web services are selfdescribing, modular business applications that expose the business logic as services over the Internet through programmable interface and where IP can be used finding ways to subscribe and invokes those services in the fixed wired network. Same way Mobile Web services are defined as self-contained modular applications that are defined, published and accessed across the Internet, in a wireless mobile communications environment using standard protocols. Web 
service defined by[1]: A Web service is a software system designed to support interoperable machine-to-machine interaction over a network. It has an interface described in a machine understandable format (specifically WSDL). Other systems interact with the Web service in a manner prescribed by its description using SOAP messages, typically conveyed using HTTP with an XML serialization in conjunction with other Web-related standards. The W3C [1] also states that two major classes of Web services: REST-compliant Web services, in which the primary purpose of the service is to manipulate XML representations of Web resources using a uniform set of "stateless" operations; and arbitrary Web services, in which the service may expose an arbitrary set of operations.

Provisioning web services on mobile smart phone is a tedious task, but mobile phones are resource constrained device, but unbelievable development in its capabilities, so it is possible to deploy web services on smart mobile phones. Still, these devices have many resource constraints or limitations like small display screen area, less battery capacity, less processing power, less memory addressing capacity, frequent disconnection of wireless network, mobility of mobile host compared to high-end fixed wired servers. While designing framework for web service provisioning should consider these resource constraints of mobile and other challenges to address the problems. However, solution for this is a lightweight hybrid framework to reduce the processing and communication overhead of a mobile host to improve the performance and reduce the battery power consumption of a mobile host. It is still in early stages to investigate effective, reliable and satisfy the need of common user to investigate lightweight framework for mobile hosted web service provisioning. There are lots of challenges to provisioning web services in the cellular domain on resource constrained mobile devices. These challenges are reducing resource consumption, mobility, security, scalability, dynamic operating environment and battery consumption.

There are several reasons for hosting a web services on mobile phone to:

- Smartphones always with user, he/she can maintain/update web service anytime anywhere

- If any updates/modification in web service, it is not affecting the client

- Client users can access the web service in an emergency situation if health related web services are available

- User get information on figure touch

- The developer can test locally, client web applications without having to consume network access

The paper is organized as follows: section 2 describes the related work of mobile web service provisioning, section 3 gives overview of mobile web service provisioning, section 4 describes extended hybrid framework REST based mobile web service provisioning of Mobile Host. Section IV gives the details of experiment setup and performance testing of the mobile host, section $\mathrm{V}$ concludes the paper and highlights the future research direction.

\section{RELATED WORK}

Provisioning web services on the resource constrained mobile devices is started from last decade. Over the past couple of years, researchers have proposed different architectures and frameworks for providing Web services from mobile smart phone. These research studies showed the feasibility and analyses the performance of a mobile host for simple web services in a controlled environment. Most of the research described mobile web service provisioning architectures and frameworks based on SOAP protocol, but very less research work for REST based mobile web service provisioning. Each one of these approaches addresses and deals with certain challenges 
facing mobile service provisioning such as reachability, reliability, and scalability. Most of the current mobile Web services provisioning architectures are immature and still in the early stages or for only a controlled environment. Also not address most important issue such as battery power consumption.

Web services access from mobile devices web server started before one decade back in 2002 with the Personal Server [3], a concept that enables people to store and access data and applications hosted on their mobile devices through convenient interfaces found nearby such as public display monitors, information kiosks, and other computers. The concept of mobile web service provisioning is not new and has been in the ground for some time. Srirama et al. proposed the concept of Mobile Host [4] in 2006 where the mobile device acts as service provider based on the SOAP protocol. Mobile Host enables seamless integration of user specific web services to the enterprise by following web service standards, also on the radio link and via resources constrained smart phones. Moreover Mobile Host fosters the new generation of ubiquitous, and context-aware applications, enabling the consumption of web services anywhere at any time, any place from the smart phones. Then the same author proposed mobile web service provisioning mediation framework in [5] as an intermediary between the web services and mobile host. In 2009 Kim and Lee [6] proposed a lightweight framework that hosts web services on mobile devices and supports service migration. In 2011 Asif and Majumdar [7] discussed partitioning frameworks for mobile web service provisioning.

The above discussed approaches focus on hosting simple web service application hosting on mobile devices. Some researchers [8],[9] and [10] has explored the usability of mobile web services in peer-to-peer environment. Mobile web service partitioning framework was proposed by [11]. Author investigated application partitioning algorithms for web service partitioning so that part of a web service can be executed on remote computing node to reduce the burden on mobile host.

Sheng [15] point out, there is a significant difference between things that work and things that work well. In this paper author propose an on demand replication approach on idle potential providers (ex. idle or less-loaded mobile devices in $\mathrm{P} 2 \mathrm{P}$ architecture) for robust Web services provisioning. According to their approach, the service provider has to maintain a ready-to-deploy (a bundle that contains all the necessary files) version of the Web service for different platforms.To accommodate more execution needs, a Web service manager can seek, on-demand, for a potential service host (from a pool of Web service hosts) to deploy the service on, if the invocation requests exceed a certain limit or to guarantee a particular performance or availability. In [16] author presented RobP2P architecure, It constructs a robust architecture for mobile P2P networks and efficiently maintain the network state. Author describes super-peer selection protocol based on an aggregate utility function that takes into account peers' capability and context. Author also presented an agile scheme through which super-peers delegate their responsibilities to more powerful and stable joining or existing peers. In result analysis showed that the RobP2P is efficient, less prone to failure, and generates lower overhead traffic, while reliably maintaining the consistency of network state.

In [17] 2013 IBM Implemented CICS JSON Web Services for Mobile Applications. In this IBM describes the existing and new aspects of IBM CICS Transaction Server that allow you to move your CICS applications, and business, into the mobile space. In this solution guide discusses an overview of how to connect mobile devices to IBM CICS Transaction Server, to use existing enterprise services that are hosted on CICS, or to develop services that support new lines of business (LOB). 
As per our previous preliminary comparative study [12] and Android Mobile Host performance evaluation of SOAP and REST [13], it showed that REST is a more suitable architecture for provisioning web services on mobile smart phones. The RESTful approach enhances the performance of mobile Web services also described by many researchers. Smart phone prices are reduced and easily available the Internet connection in very cheap rate, usability of Smart phone increased. While considering today's scenario of Smart phones is becoming more and more-smart in terms of processing power or more capable processor, memory addressing capability and advancement in mobile wireless technologies and web technologies. Due to this, mobile computing is becoming more and more popular for pervasive computing. Deploying web services on smart phone it is today need, because anyone can access mobile hosted web services any time anywhere and managing mobile hosted web services is also very easy.

\section{Basic of Mobile Web Service Provisioning}

Mobile web service provisioning is art to allow programmers to deploy, discover, publish and execute of web services in wireless environment using standard protocols and without disturbing basic functionality of smart phone use mobile phone as web service provider. Mobile web service provisioning can be classified based on architecture is used to providing web service access to end-user.

1. SOA (Service Oriented Architecture) or SOAP based Mobile web service provisioning

2. ROA (Resource Oriented Architecture) or REST based Mobile web service provisioning.

\subsection{SOAP Based Mobile Web Service Provisioning}

SOAP based web services are also called as big web services. This kind of web services uses service oriented architecture and populating contents they use SOAP (Simple Object Access Protocol) protocol to communicate between the client interface and web service provider. SOAPbased Web services are used RPC-like interactions while communicating with remote web service consumer. SOAP based service provider and web service client need to follow service syntax and data types. It uses Web Services Description Language (WSDL) for describing functionality offered by deploying web service and Universal Description Discovery and Integration (UDDI) is used as a central registry for deploying web services. The main functions of UDDI are to manage, maintain and provide reference to the WSDL file. For interaction between the service provider and service requester XML is used. This type of web service provisioning has only one end point communication address and which is defined by the single URI. This address is stored in UDDI registry and used by the web service to receive SOAP request. SOAP based web services require discovery phase but REST based web services are not requires discovery phase. The Web service itself is responsible for handling all the communications directed to its internal operations.

SOAP based web service provisioning architecture has three actors: Web Service Requestor (Client), Web Service Provider (Mobile Host/ Server) and Web Service Registry. The mobile web service provider publishes its Web Services with the service registry. The service requestor client (mobile, PDA, PC or Laptop) searches ("Find") the UDDI registry for the services, and the UDDI compatible service registry refers the respective WSDL. The service requestor client accesses the described Web Service, using SOAP is shown in figure 1. 


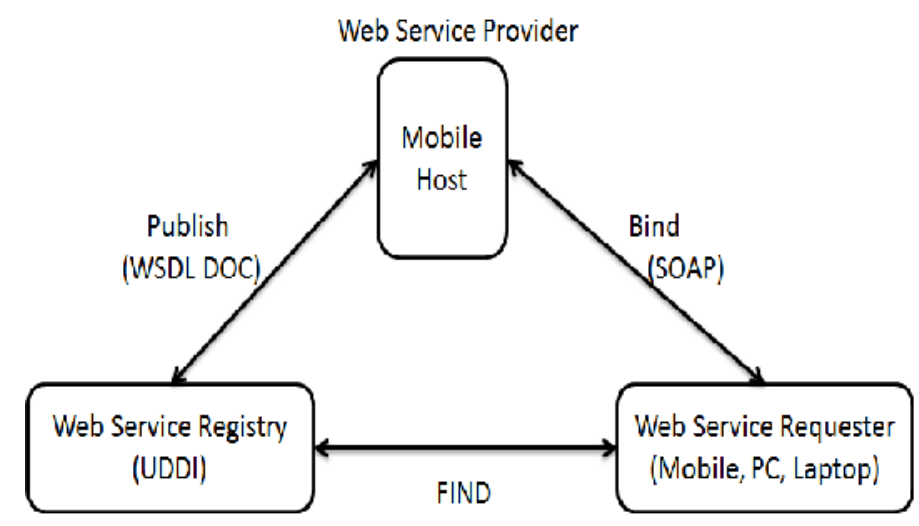

Figure 1. SOAP based Mobile web service provisioning

\section{B. REST Based Mobile Web Service Provisioning}

SOAP is designed for a fixed network environment where high end servers are used, but REST is alternative to SOAP for the fixed network environment as well as mobile network environment and resource constrained devices. REpresentational State Transfer (REST) is also called Resource Oriented Architecture (ROA), is a style of software architecture that relies on the fact that any resource (such as Web services) can be identified by their URLs. REST is an architectural style [2] defined in his dissertation and it is derived from the Web, and its purpose is to assemble the fundamental design principles that enable the greater scalability, growth and success of the Web services on the web.

REST is initially used for publishing hypermedia documents but later it is becoming a common scheme for realizing Web services on fixed network as well as a mobile network. REST follows a different philosophy than SOAP by focusing on data instead of operations and also to provide resource friendly alternative to SOAP. REST based web services are tightly coupled with the HTTP protocol however and compromise their flexibility and portability. RESTful Web services gained much attention from the Web community due to their simplicity and scalability. Major Web service providers such as Google, Amazon, Yahoo, and eBay adopted the RESTful Web services approach in their offered Web services. RESTful Web services use unique URIs for identification of resources. These resources are accessed and manipulated using a set of uniform methods GET, POST, PUT and DELETE.

REST based web service provisioning architecture has two components: Web Service Requestor (Client), Web Service Provider (Mobile Host). In this web client directly send HTTP request to host and host parse the request and web servlet generate response, and then response sends back to the client using the same protocol. REST based web service architecture shown in above figure 2 . 


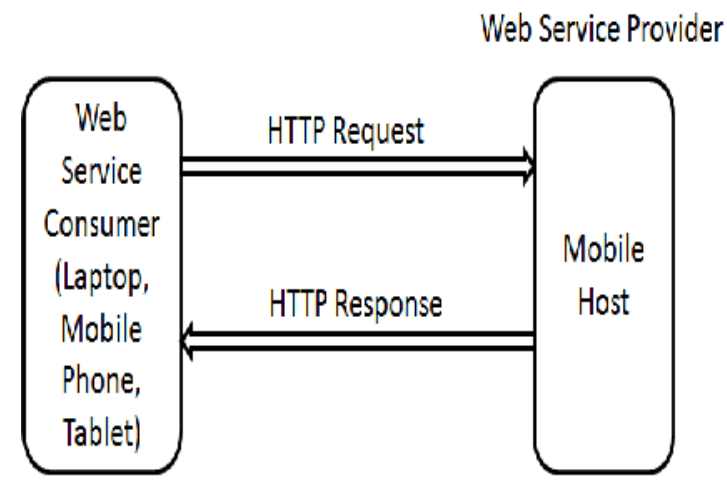

Figure 2. REST based Mobile web service provisioning

\section{Mobile Host}

Nowadays, smart phones are used to access information, emails, map service, etc, of other than voice calls. Smart phones are using a wide variety of services like location based services, mobile cloud services, mobile web services etc, from different providers. As latest developments in smart mobile phones, software technologies, and wireless infrastructure, a new type of service provider comes in mobile phone users themselves with the help of Mobile Host. The latest version of Mobile Host was developed for Android OS taking into account that it has the biggest market share in smart phone operating systems. Along with upgrading to Android OS other improvements were made. SOAP architecture was replaced with REST architecture, Fuzzy controller for monitoring resource of the hybrid framework, SMS based web service access for alternative to HTTP was used as module system and services platform to handle components life cycle without reboot and of course when talking about Android applications development, Android Software Development Kit was used when establishing Server Socket communication so HTTP requests could be handled.

There are two different mechanisms for interacting with web services, SOAP and REST. As an improvement, SOAP architecture was replaced with the REST architecture in a Mobile Host for Android. For providing services from mobile devices, REST is the logical option due to its cacheable and stateless characteristics. The architecture of the Mobile Host for Android OS is shown in Figure 3.

Mobile Host for open source Android OS is designed to follow the RESTful architecture. REST is an architecture in which the key resources (entities, collections, services, etc.) are identified by its own URI. The standard methods are mapped to resource-specific semantics. All resources, implement the same uniform interface. Mobile Host accepts web service requests through HTTP or SMS Messaging. When the HTTP / SMS interface receives web service request then it is passed to Request Handler which does the parsing of the request and its parameters. The Request is passed to the Request Resolver when the REST handler has parsed the request. Request Resolver then communicates with the HTTP manager and SMS manager. Then HTTP and SMS manager, send to service selector. Proposed service selector algorithm with output of fuzzy controller to select the web service and generates the response for web clients. 


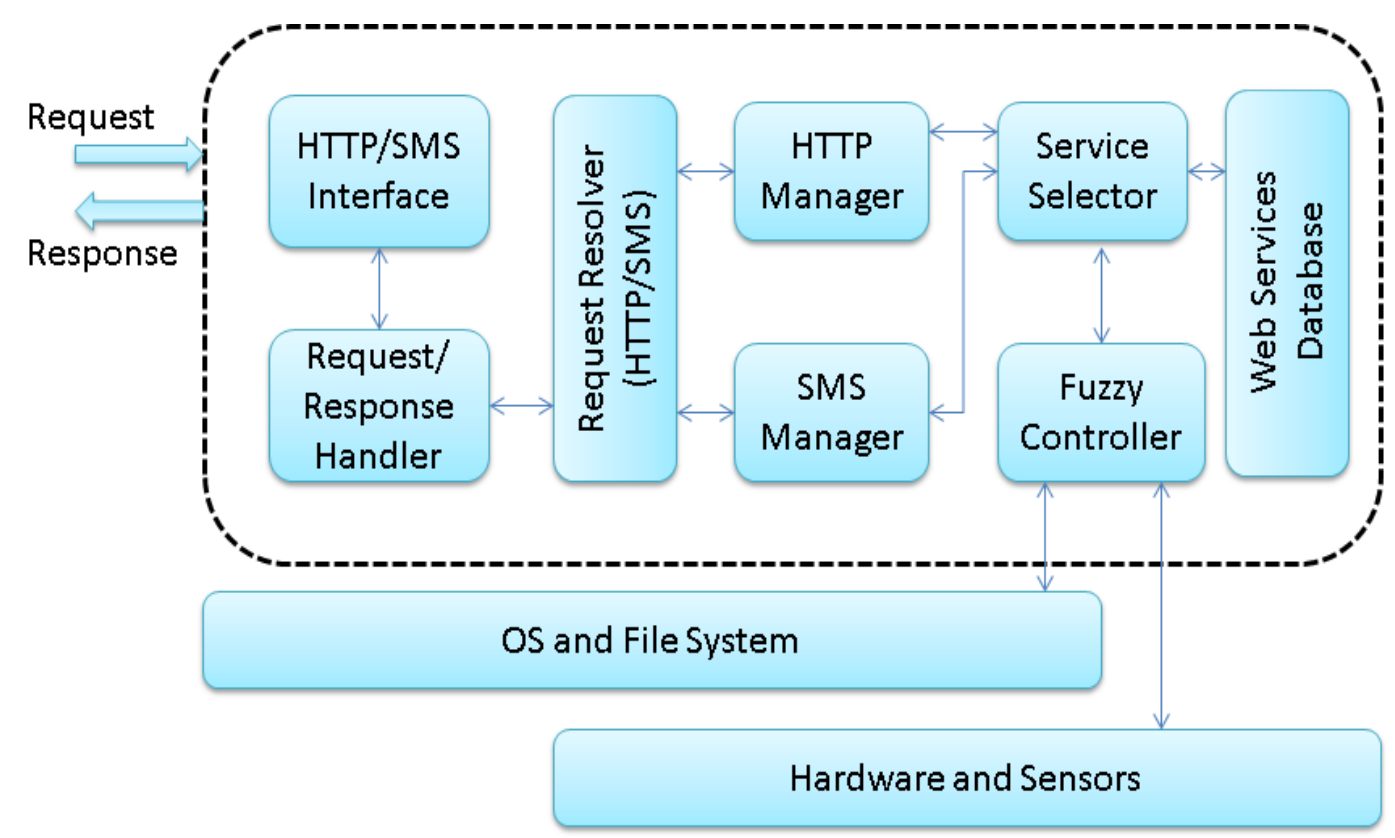

Figure 3. Architecture of the Mobile Host for Android OS

\section{Performance Analysis of Mobile Host}

Mobile Host is deployed on Samsung Galaxy S Duos S7562 Android Smartphone, 4GB internal memory, $1 \mathrm{GHz}$ Cortex-A5 processor. Performance of Mobile Host was analysed with Hospital and Blood bank Search web service. Deployed web services are based on REST architecture style using corresponding URL and provide access to detailed information related to the particular service.

For performing controlled web service architecture, preferred jfuzzylite rather that jfuzzylogic because of some advandvantages that jfuzzylite has. Comparatively Size of jfuzzylite is very small than jfuzzylogic. Size of jfuzzylite is approximately $300 \mathrm{~KB}$ which makes it ideal for the Android platform and size of jfuzzylogic is $2.94 \mathrm{MB}$.

jfuzzylite is a cross-platform, free and open-source fuzzy logic control library programmed entirely in Java. Its goal is to allow to easily create fuzzy logic controllers in a few steps utilizing object-oriented programming without requiring any third-party libraries. jfuzzylite is the equivalent of the fuzzylite library for Java and Android platforms introduced by [13].

Fuzzy logic has several features that make it suitable for developing controlled web service architecture on mobile device. One of the most significant features is the flexible decision making from the data parameters. Fuzzy logic control provides a powerful alternative to traditional control algorithms that avoids the consumption of a large amount of resources like CPU and memory.

The experiments testing a GET request for getting hospital or blood bank information and POST to add information of hospital or blood bank in database. The services were developed based on the Android platform, compatible with Android 2.2 API or higher. The experiments were conducted under Wi-Fi, 2G, 3G and 4G networks. 
International Journal on Web Service Computing (IJWSC), Vol.5, No.2, June 2014

1. The tests were taken in a Wi-Fi network with upload rate of $40 \mathrm{Kbps}$ and download rate of $150 \mathrm{Kbps}$, respectively and a $2 \mathrm{G}$ network with upload rate of $15 \mathrm{Kbps}$ and download rate of $48 \mathrm{Kbps}$, a $3 \mathrm{G}$ network with upload rate of $60 \mathrm{kbps}$ and download rate $800 \mathrm{Kbps}$ and a 4G network with upload rate $700 \mathrm{Kbps}$ and download rate $2 \mathrm{Mbps}$. Figure 4 shows the 30 to 35 concurrent user supported by a mobile host without degrading the performance.

2. The tests were taken TP-Link TD-W8151N Wireless ADSL2+ Router Wi-Fi network that will allow to access and share the Internet over a large space, seamlessly.Mobile host is connected to this router. Wi-Fi network with Downstream data rates up to 24Mbps, upstream data rates up to $3.5 \mathrm{Mbps}$ and Airtel $4 \mathrm{G}$ network with upload rate upload speed is $15 \mathrm{Mbps}$ and download speed is $35 \mathrm{Mbps}$.Figure 5 shows the 150 to 200 concurrent user supported by Mobile host without degrading the performance.

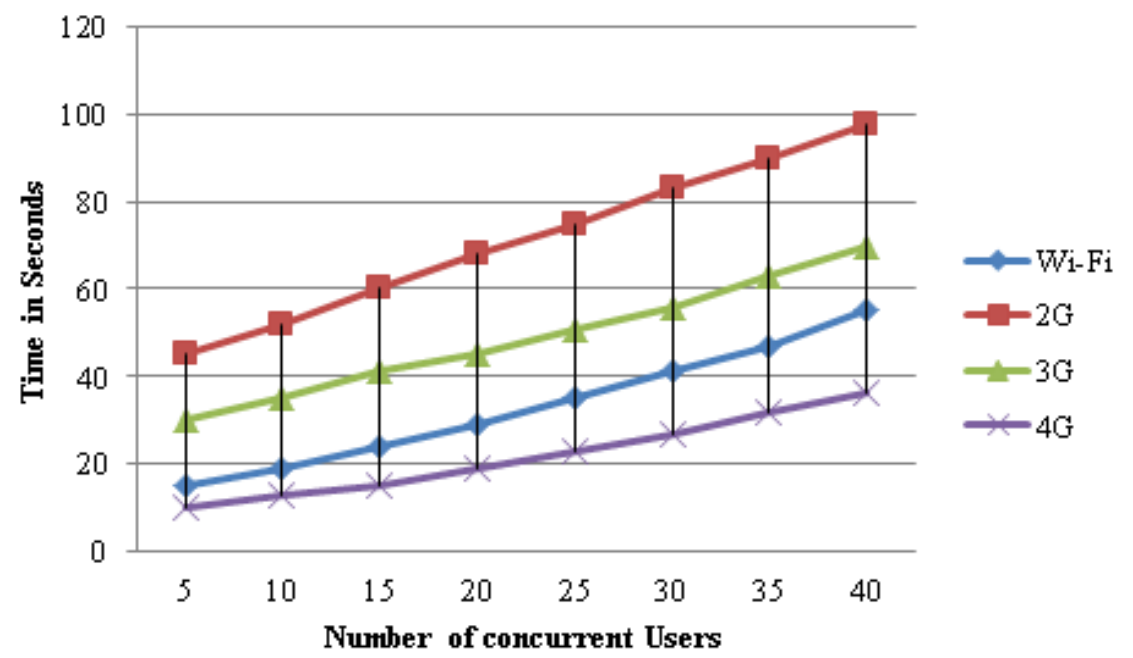

Figure 4. Mobile Host for concurrent users access

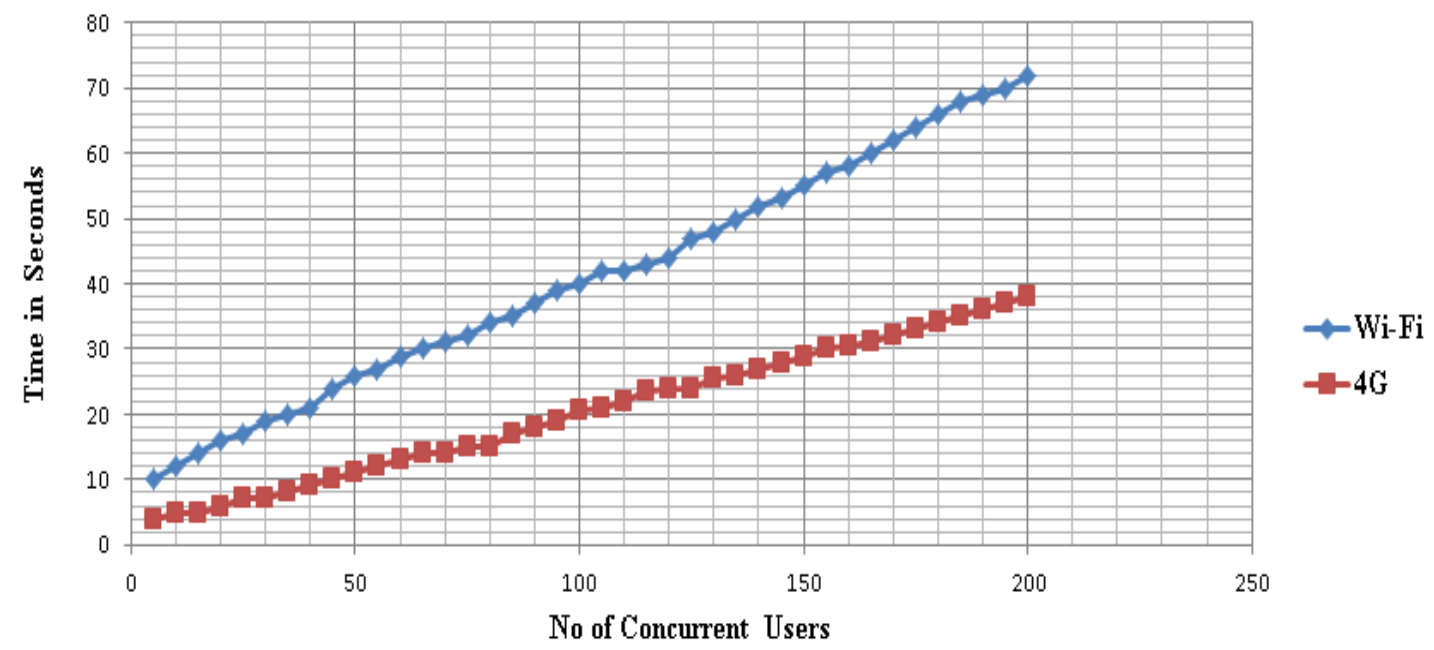

Figure 5. Mobile Host with 200 concurrent users access 
From analysis, it is observed that Mobile Host for Android can handle a more number of connections as compared to the previous researchers result.

\section{Conclusions}

Android based Mobile Host is implemented and tested successfully using REST web service provisioning concept. From analysis shows improved capabilities of today's mobile devices are supported more number of concurrent users. SMS provides alternative way to access web services and it shows that any mobile user can access web services hosted on smart phone without GPRS. From the performance testing results it is showing that the Mobile Host for Android presents reasonable performance when providing mobile web services to more users. A Web Service provider demonstrated the technical feasibility of this approach in terms of resource consumption, standard compliance, and performance. The approach truly paves scope for the client-server and distributed mobile information networks. In future mobile host will be tested for more complex web services.

\section{REFERENCES}

[1] http://www.w3.org/TR/ws-arch/ Web Services architecture.

[2] R. Fielding, "Architectural Styles and The Design of Network based Software Architectures", PhD Thesis, University of California, Irving, 2000

[3] Royu Want, Trevor Pering, Gunner Danneels, Muthu Kumar, Murali Sundar, and John Light., (2002), "The Personal Server: Changing The Way We Think About Ubiquitous Computing," In The International conference on Ubiquitous Computing, pp. 194-209.

[4] S. N. Srirama, M. Jarke, W. Prinz (2006), Mobile web service provisioning, in: AICTICIW '06: Advanced Int. Conf. on Telecommunications and Int. Conf. on Internet and Web Applications and Services, IEEE Computer Society, 2006, pp. 120-126.

[5] S. Srirama, M. Jarke and W. Prinz, (2006) "Mediation Framework for Mobile Web Service Provisioning", 10th IEEE International Enterprise Distributed Object Computing Conference workshop 2006, EDOCD '06', pp $14-21$.

[6] Y. Kim and K. Lee, (2009), "A lightweight framework for mobile web services," Computer ScienceResearch and Development, vol. 24, no. 4, pp. 199-209.

[7] M. Asif and S. Majumdar, (2011), "Partitioning frameworks for mobile web services provisioning," International Journal of Parallel, Emergent and Distributed Systems, vol. 26, no. 6, pp. 519-544.

[8] L. Pham and G. Gehlen, (2005), Realization and Performance Analysis of a SOAP Server for Mobile Devices, In the Proceedings of the Eleventh European Wireless Conference,Vol. 2, Nicosia, Cyprus, 20-27

[9] G. Gehlen and L. Pham, (2005), Mobile Web Services for Peer-to-Peer Applications, In the Proceedings of the Second IEEE Consumer Communications and Networking Conference (CCNC'05), Las Vegas, NV, U.S.A., 427-433.

[10] F. Aijaz, S. Adeli and B. Walke, (2008), Middleware for Communication and Deployment of Time Independent Mobile Web Services, In the Proceedings of the IEEE International Conference on Web Services (ICWS'08), Hawaii, HI, U.S.A., 797-800.

[11] M. Hassan, (2009), Mobile Web Service Provisioning in Peer to Peer Environments, IEEE International Conference on Service Oriented Computing and Applications, 1-4.

[12] Wagh Kishor, Ravindra Thool, (2012), A comparative study of soap vs rest web services provisioning techniques for mobile host, Journal of Information Engineering and Applications Vol. 2, Issue 5, 1216.

[13] Kishor S Wagh, R C Thool, (2013), Web Service Provisioning on Android Mobile Host, International Journal of Computer Applications, Vol. 81 No14, Published by Foundation of Computer Science, New York, USA., 5-11.

[14] E. H. Mamdani and S. Assilian, (1975), An experiment in linguistic synthesis with a fuzzy logic controller, International Journal of Man-Machine Studies, vol. 7, no. 1, 1-13. 
[15] Q. Z. Sheng, Z. Maamar, J. Yu, and A. H. H. Ngu, (2009), Information Systems: Modeling, Development, and Integration, ch. Robust Web Services Provisioning through On-Demand Replication, Springer Berlin Heidelberg, 4-16

[16] Khalid Elgazzar, Walid Ibrahim, Sharief Oteafy, Hossam S. Hassanein, (2013), RobP2P: A Robust Architecture for Resource Sharing in Mobile Peer-to-Peer Networks, The 4th International Conference on Ambient Systems, Networks and Technologies (ANT 2013), 356-363.

[17] http://www.redbooks.ibm.com/abstracts/tips1066.html?Open [Assced on 26 June 2014]

\section{Authors}

Kishor S. Wagh.received the BE degree in Computer Engineering from North Maharashtra niversity, Jalgaon, Maharashtra, India, in 1996 andthe ME in computer Engineering in 2006 from PICT, University Pune. He is Assistant Professor with the department of Computer Engineering, Marathwada Mitra Manadal's Institute of Technology, Pune. Currently pursuing his $\mathrm{PhD}$ in Computer Science and Engineering from SRTM University, Nanded. His research area includes Web Services, wired and wireless networks and could computing. He is member of CSI and Life member of ISTE.waghks@mmit.edu.in

Dr. Ravindra C. Thool received his BE, ME and Ph.D. in Electronics from SGGS College of Engineering \& Technology, Nanded, Maharashtra, India, in 1986, 1991 and 2003 respectively. He is professor in Information Technology, in the same organizatio $\mathrm{n}$. His research area includes Computer Vision, Image processing, Wireless networking, Mobile computing and multimedia information systems. He has published several research papers in refereed journals and professional conference proceedings. He is member of IEEE and Life member of ISTE. rcthhol@sggs.ac.in
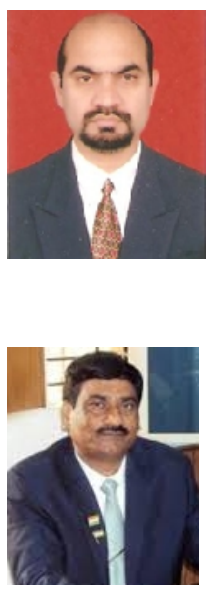